\title{
Evaluation of anti-leishmanial effects of killed Leishmania vaccine with BCG adjuvant in BALB/c mice infected with Leishmania major MRHO/IR/75/ER
}

\author{
Hossein Nahrevanian ${ }^{1}$, Seyedeh Parisa Jafary ${ }^{2}$, Sara Nemati ${ }^{1}$, Mahin Farahmand ${ }^{1}$ and Eskandar Omidinia ${ }^{3}$ \\ ${ }^{1}$ Department of Parasitology, Pasteur Institute of Iran, Tehran, Iran; \\ ${ }^{2}$ Islamic Azad University of Oloum Tahghighat, Tehran, Iran; \\ ${ }^{3}$ Department of Biochemistry, Pasteur Institute of Iran, Tehran, Iran
}

\begin{abstract}
There are many strategies to control leishmaniasis, but majority of them are inadequate. Killed Leishmania vaccine (KLV) has been applied for its immunogenicity in human and mouse model. Bacillus Calmette-Guerin (BCG) as adjuvant is an immunemodulator inducing humoral and cellular immune responses during zoonotic cutaneous leishmaniasis (ZCL). Both KLV and BCG have been applied for their immune responses in hosts for controlling leishmaniasis. In this study, KLV and BCG were applied to inhibit replication and visceralization of Leishmania major in BALB/c mice. Mice were injected with KLV and BCG, followed by infection with promastigotes of L. major. Six weeks after infection, a small nodule appeared, which was followed by development of a large lesion and visceralization. Effects of KLV and BCG, physiopathological changes, lesion size, delay of lesion formation, proliferation of amastigotes inside macrophages and detection of amastigotes in target organs were studied. Results showed that the KLV had anti-leishmanial activity by reducing lesion size on late infection. In KLV and BCG group, the average number of amastigotes in macrophages was lower than in other groups. Significant reductions in number of amastigotes in both spleen and lymph node were observed, indicating lower visceralization of Leishmania parasites in these target organs. No significant changes were presented in body weights, survival rates and degrees of splenomegaly in test group. It can be concluded that application of KLV and BCG had acceptable efficacy in reduction of skin lesions size and proliferation of parasites, even though a few side-effects were observed. It is indicated that KLV/BSG may have ability to modulate host immune responses against Leishmania parasites and to reduce pathophysiology of the disease during infection.
\end{abstract}

Keywords: vaccine, KLV, adjuvant, Leishmania major, immunization, Iran

Leishmaniasis is one of the most important infectious diseases worldwide, causing a spectrum of manifestations ranging from asymptomatic infections and mild self-healing cutaneous disease to severe non-healing diffuse cutaneous (CL) and visceral leishmaniasis (VL) (Manson-Bahr and Apted 1983). Cutaneous form of leishmaniasis caused by Leishmania major (Leishman, 1903) (NCBI ID: 5664) and L. tropica (Wright, 1903) (NCBI ID: 5666), which is endemic in more than 80 countries, with $90 \%$ cases reported in six countries only, namely Afghanistan, Brazil, Iran, Peru, Saudi Arabia and Syria. CL is an important public health problem in many parts of Iran (Klaus et al. 1999).

Lesions are characterized by development of nodules, which progress to ulcerative lesions. Immune responses during leishmaniasis include antibodies, cytokines, immune cells, mediators and acute phase proteins (Pearson et al. 1983). In CL, various phagocytes predominant in the skin, including neutrophils, macrophages and dendritic cells, play distinct roles for the host's immune responses (Scott 2005).
The main line of treatment in leishmaniasis is pentavalent antimonials such as meglumine antimoniate or Glucantime. These antimonials were first introduced in 1945 and remain effective treatments for some forms of leishmaniasis. However, long periods of parenteral administration, variable efficacy against $\mathrm{CL}$ and VL, some side effects and the emergence of significant resistance are all factors that limit the usefulness of these drugs (Roberts et al. 1998). Vector control is insufficient and complicated due to the diverse ecology of different species of sand fly vectors and animal reservoirs. In addition, existing treatments are expensive and effective vaccines against leishmaniasis currently do not exist (Noazin et al. 2009). The high prevalence of leishmaniasis and the resistance to conventional drugs demonstrate the need for novel targets with lower toxicity and more efficiency (UNDP/World Bank/WHO 1997).

The development of an affordable vaccine is considered the only cost-effective means to control this type of leishmaniasis. Moreover, vaccination with parasites (leishmanization) has been used as an immunisation 
method in Iran (Nadim and Javadian 1988), Israel (Green et al. 1983) and former Soviet republics Turkmenistan and Uzbekistan (Kobets et al. 2012), but was abandoned because of the occurrence of a small proportion of chronic cases, which required long time treatment. Vaccination (leishmanization) with live parasites was practiced in the Middle East, but was later replaced with the use of killed Leishmania parasites for the protection against CL in both the Old and New World (Mohebali et al. 1995). The autoclave-killed L. major was used for leishmanization of about 2 million individuals in the 1980s in Iran and safety and immunogenicity of this vaccine was shown (Mohebali et al. 1995). In addition, positive results have been reported by successful vaccination against VL using intradermal inoculation of alum-precipitated autoclaved L. major (ALM) or L. donovani (ALD) with Bacillus Calmette-Guerin (BCG) in Indian langurs (Garg and Dube 2006). The significant efficacies of attenuated $L$. major vaccine were also demonstrated for a Rhesus monkey model of the human disease (Amaral et al. 2002) and in dogs vaccinated with autoclaved $L$. major promastigotes (ALM) plus BCG in Iran (Mohebali et al. 2004). In addition, the pioneer use of killed Leishmania vaccine (KLV) plus BCG for immunotherapy was applied in Venezuela with considerable success (Convit et al. 1987). Application of KLV without BCG was reported to be safe in Brazil with limited protection (Mayrink et al. 1979, 1985, Antunes et al. 1986). Later, KLV plus BCG were successfully used for immunotherapy in localized cutaneous leishmaniasis in Venezuela (Convit et al. 1987).

Mycobacterium bovis BCG as adjuvant is inducing humoral and cellular immune responses during leishmaniasis. The ability of the two recombinant BCG strains to induce protective immunity against a challenge with L. major amastigotes was evaluated after vaccination of susceptible BALB/c and resistant $\mathrm{C} 57 \mathrm{BL} / 6$ mice. They showed significant protection against a challenge with L. major in BALB/c-immunized mice (Nateghi Rostami et al. 2010, Smrkovski and Larson 1977b, Latifynia and Mohaghegh Hazrati 2008). Research on a vaccine against leishmaniasis in Iran began with the preparation of a KLV under manufacturing practices at the Razi Serum and Vaccine Research Institute (RSVRI), Hesarak, Karaj, Iran. KLV has been applied for its immunogenicity in both human and mouse model (Abdelhak et al. 1995). Inoculation of BCG was found to be both prophylactic and therapeutic in BALB/c mice against challenge with amastigotes of Leishmania (Smrkovski and Larson 1977a, b, Latifynia and Mohaghegh Hazrati 2008).

The objective of this study was to evaluate the protective effects of KLV with BCG on L. major infection in $\mathrm{BALB} / \mathrm{c}$ mice. The inhibition of visceralization, differences of lesion sizes, duration of disease, rate of parasite proliferation inside macrophages and survival rate were studied to evaluate the efficacy of BCG + KLV.

\section{MATERIALS AND METHODS}

Animals. Female inbred SPF BALB/c mice 4-6 weeks old (supplied by the Karaj Laboratory Animal Unit, Pasteur Institute of Iran) were used in this study. The $18.2 \pm 1.3 \mathrm{~g}$ (Mean \pm Standard Error, SE) mice were housed at room temperature $\left(20-23^{\circ} \mathrm{C}\right)$ on a $12 \mathrm{~h}$ light and $12 \mathrm{~h}$ dark cycle, with unlimited access to food and tap water. Experiments with animals were done according to the ethical standards and measures taken to protect animals from pain or discomfort. It has been approved by Ethical Committee of the Pasteur Institute of Iran, in which the work was done.

Leishmania parasite. Leishmania major MRHO/IR/75/ER used in this study was the standard Iranian strain. The infectivity of the parasites was maintained by regular passage in susceptible $\mathrm{BALB} / \mathrm{c}$ mice. The parasites were cultured in the RPMI 1640 medium supplemented with $10 \%$ Fetal Bovine Serum (FBS), $292 \mu \mathrm{g} / \mathrm{ml} \mathrm{L}$-glutamine and $4.5 \mathrm{mg} / \mathrm{ml}$ glucose (all supplied by Sigma Aldrich). Under these culture conditions, the stationary phase of parasite growth was obtained in six days (Nahrevanian et al. 2007).

Infection of BALB/c mice with $L$. major. Promastigotes of L. major were harvested from culture media, counted and used to infect BALB/c mice. The base of the tail was injected intradermally with inoculums of $2 \times 10^{6}$ promastigotes. The animal experiments were performed once in five groups $(\mathrm{n}=10$ mice/ group) considering time, budget and long-period monitoring of animals. The Leishmania infection was carried out in experimental animals and terminated at week 16 after injection.

Vaccine. The KLV used in this study was autoclaved L. major promastigotes prepared by RSVRI, Hesarak, Karaj, Iran and kindly donated by Department of Immunology, Pasteur Institute of Iran, Tehran, Iran. The vial content of vaccine was diluted with the normal saline to have a volume of $10 \mathrm{ml}$. Each mouse in KLV and KLV+BCG test groups received $100 \mu \mathrm{l}$ of this solution per injection.

BCG. The lyophilized BCG vaccine (Mycobacterium bovis, Bacillus Calmette Guerin) was prepared in the Pasteur Institute of Iran and stored at $4{ }^{\circ} \mathrm{C}$ until use. Just before injection, it was suspended in diluted solution SSI solvent $\left(125 \mathrm{mg} \mathrm{MgSO}_{4}, 125 \mathrm{mg}\right.$ $\mathrm{K}_{2} \mathrm{HPO}_{4}, 1 \mathrm{mg}$ L-aspargine, $\mathrm{H}_{2} \mathrm{O}, 12.5 \mathrm{mg} \mathrm{Fe}$ ammonium citrate, $18.4 \mathrm{mg} 85 \%$ glycerol, $0.5 \mathrm{mg}$ citric acid, $1 \mathrm{ml} \mathrm{H}_{2} \mathrm{O}$ for injection/manufacturer's insert) with $0.1 \mathrm{mg}$ BCG. All doses were injected intradermally in susceptible BALB/c mice.

Experiments and groups. Animals used in this experiment were BALB/c mice, divided into five groups (10 mice/group) including Group 1 (Naive), Group 2 (KLV), Group 3 (BCG), Group $4(\mathrm{KLV}+\mathrm{BCG})$ and Group 5 (Control L. major). One week after the first KLV and BCG injections, the second phase as booster with the same dose was injected subcutaneously (s.c.) in abdominal area. The challenge of mice within groups 2 to 5 was carried out one hour after the second injection of KLV and BCG s.c. at the base of the tail by inoculation of $300 \mu \mathrm{l}$ containing $10^{6} \mathrm{~L}$. major promastigotes obtained from stationary phase of cultivation tubes.

Measurement of lesion size. Lesion size was measured at every other week after inoculation in millimetres $(\mathrm{mm})$ by a digital caliper (Chuan Brand, China) in two diameters (D and d) at right angles to each other and the size $(\mathrm{mm})$ was determined according to the formula: $\mathrm{S}=(\mathrm{D}+\mathrm{d})$ divided by 2 (Erel et al. 1999). 
Microscopical examinations and smear preparation. The clinical diagnosis was confirmed by laboratory demonstration of the parasite in the lesions by making stained smears at the end of the experimental period. Lesions were cleaned with ethanol and punctured at the margins with a sterile lancet and exudation material was smeared. Impression smears were prepared from the liver, spleen and lymph nodes by placing a small piece of tissue between two glass slides and pushing them in different directions. The smears were dried in air, fixed by methanol and stained with Giemsa for detection of amastigotes under light microscopy (El-On et al. 1986).

Measurement of proliferation of amastigotes. The proliferation of parasite was evaluated by counting amastigotes inside macrophages on Giemsa stained lesion smears at the end of the experimental period. Five random macrophages were selected and counted; mean percentages were calculated as indicators for the degree of proliferation of amastigotes inside each macrophage (Nahrevanian et al. 2007).

Assessment of degree of hepatosplenomegaly. Entire livers and spleens were removed post mortem at the end of the experimental period from mice after induction of terminal general anaesthesia by inhalation of diethyl ether (Sigma Aldrich). Organ wet weights were measured, expressed as ratio to body weight and compared with controls as indices for degree of hepatosplenomegaly.

Measurement of survival rate and body weight. Survival rate was presented as percentage of surviving experimental mice at every other week after inoculation; the significance of differences was compared with concurrent appropriate vehicle-treated Leishmania and control groups. Body weight was measured initially and at different time of experiment using a top pan balance (Ohasus Scale Corp., USA).

Statistical analysis. Values are presented as the mean \pm standard error (SE) for groups of $n$ samples. The significance of differences was determined by One-Way Analysis of Variances (ANOVA) and Student's $t$-test using Graph Pad Prism Software (Graph Pad, San Diego, California, USA) and Microsoft Excel 2007.

\section{RESULTS}

Healing of cutaneous lesions was studied by measurement of lesion sizes in both control and test groups. Saline had no effects on lesion size, but the group that received only KLV showed anti-leishmanial activity, as this compound reduced the lesion size significantly $(\mathrm{P}<0.05)$ after 13 weeks of infection. There was no significant indication of BCG to limit lesion size in CL as presented in experimental groups (Fig. 1). The number of amastigotes in macrophages (MQ) of lesion samples represented a significant change, which is indicated in the group receiving $\mathrm{KLV}+\mathrm{BCG}$. In this group, the average number was lower than in the other groups (Fig. 2).

When considering visceralization of Leishmania parasite in target organs, it represented significant reductions in the number of amastigotes in both spleen $(\mathrm{P}<0.01)$ and lymph node $(\mathrm{P}<0.001)$ tissue smears. However, there was no significant difference based on parasite percentage in smears of liver tissue (Fig. 3). No significant changes were observed in body weights, survival rates and de-

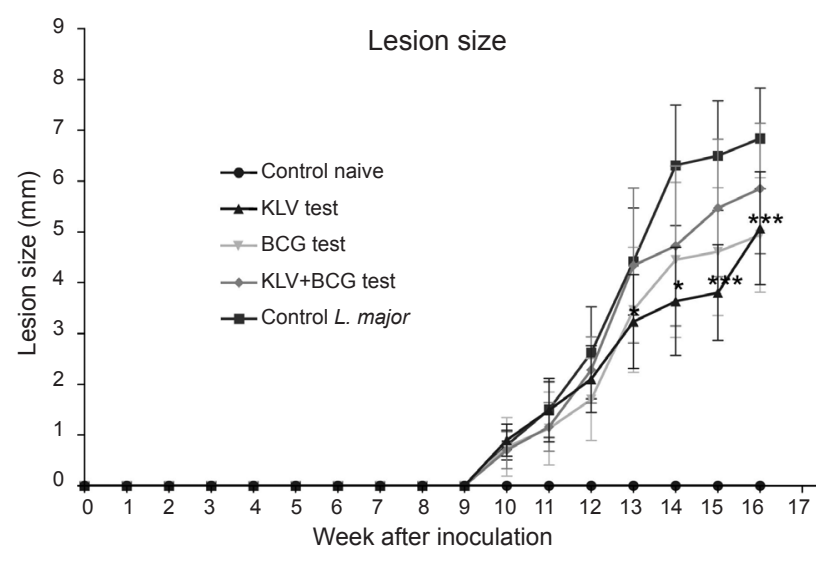

Fig. 1. Influence of vaccine/adjuvant on skin lesion development. Progress of lesion sizes of cutaneous leishmaniasis in groups of $\mathrm{BALB} / \mathrm{c}$ mice infected with Leishmania major. The results expressed as mean \pm SE. Significance of differences $(\star \mathrm{P}<0.05$ and $\star \star \star \mathrm{P}<0.001)$ were determined by ANOVA test using Graph Pad Prism ( $\mathrm{n}=10$ mice/group).

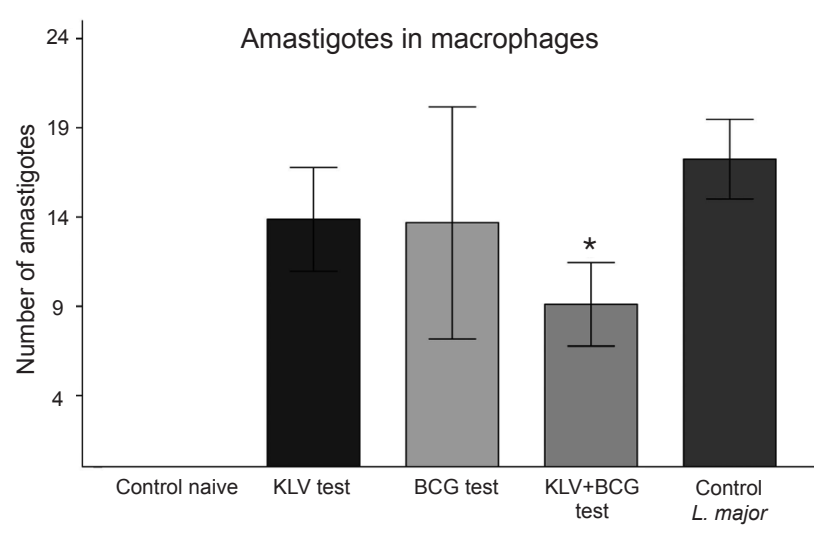

Fig. 2. Percentage of proliferation of amastigotes of Leishmania major inside mouse macrophages in positive lesion smears. Proliferation of amastigotes inside macrophages was counted on Giemsa stained lesion smears at the end of the experimental period. The results expressed as mean \pm SE. Significance of difference $(* \mathrm{P}<0.05)$ was determined by ANOVA test using Graph Pad Prism ( $\mathrm{n}=10$ mice/group).

grees of splenomegaly in test group compared with those in controls. Degree of hepatomegaly presented a significant change $(\mathrm{P}<0.001)$ in the group that received only $\mathrm{BCG}$ in comparison with other groups (Fig. 4).

\section{DISCUSSION}

The group that received only KLV showed anti-leishmanial activity because this compound reduced the lesion size significantly after week 13 of infection. In a previous study of the present authors, application of KLV solely or with Imiquimod (IMQ) as adjuvant resulted in no changes in lesion size variations in BALB/c mice infected with the same Leishmania major strain (Salehizadeh et al. 2011). Evidence exists that BCG alone gives some protection against experimental leishmaniasis in mice (Smrkovski and Larson 

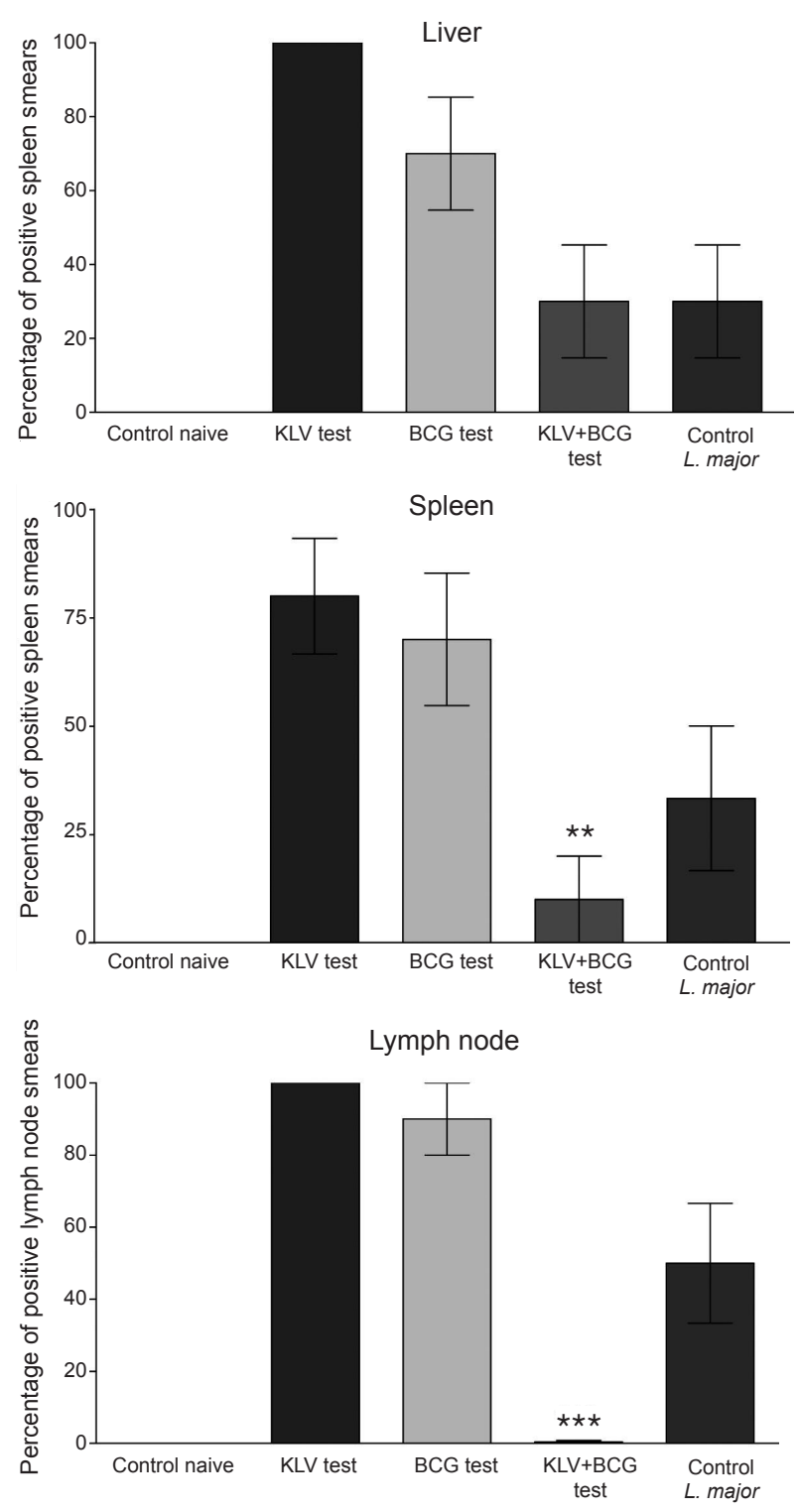

Fig. 3. Percentage of Leishmania visceralization in target organs. Two impression smears from each tissue/mice, were prepared from the liver, spleen and lymph nodes. The results expressed as mean $\pm \mathrm{SE}$. Significance of differences $(* \star \mathrm{P}<0.01$ and $\star \star \star \mathrm{P}<0.001$ ) were determined by ANOVA test using Graph Pad Prism ( $\mathrm{n}=10$ mice/group).

1977b, Weintraub and Weinbaum 1977). In another study evaluating effect of KLV plus BCG in healthy individuals in Venezuela, it was found that the vaccine resulted in conversion in Delayed Type Hypersensitivity (DTH) of the vaccinated subjects (Castes et al. 1994). A similar study using the autoclaved L. major (ALM) plus BCG in a $L$. major endemic focus in Iran showed a greater degree of immunogenicity of the vaccine over the BCG alone, and recommended booster doses with other adjutants (Sharifi et al. 1998).

The number of amastigotes in MQ represented a significant reduction in group that received $\mathrm{KLV}+\mathrm{BCG}$ treatment. This is not in agreement with our previous study with similar parasite, host and methodology, in which applications of KLV and IMQ (as adjuvant) revealed no significant effects on the proliferation rate of amastigotes inside MQ (Salehizadeh et al. 2011). As in previous studies, lesions in mice given BCG before challenge with L. major were less severe and contained significantly fewer parasites than those of similarly infected control mice not given BCG (Stefani et al. 1993).

Although no variations were observed based on parasite percentage in liver smears, a significant decline in the number of amastigotes in both spleen and lymph node tissue smears as target organs was emphasized as inhibition of visceralization by KLV + BCG effects. This is in agreement with the authors' previous study, indicating an observable inhibition of visceralization using KLV + IMQ in the liver and spleen. However, IMQ solely decreased the visceralization in the lymph nodes (Salehizadeh et al. 2011). This evidence highlights the important role of vaccine (KLV) and/or adjuvant (BCG) in control of visceral form of leishmaniasis; further studies are required to discuss this concept.

One major aspect in the use of vaccines is the assessment of side effects associated with vaccination. No significant changes were observed in pathophysiological effects due to KLV and BCG including body weights, survival rates and degrees of splenomegaly, which indicated the lowest toxicity of KLV/BCG and confirms results of our previous study on KLV + IMQ (Salehizadeh et al. 2011). Physiological parameters represented no pathological consequences on the host after vaccine (KLV) and adjuvant (BCG) injections, separate or in accompany. Therefore, they can be applied due to the minimum cytotoxic effects in BALB/c model of leishmaniasis. In this study, no serious life-threatening reactions were associated with the vaccine and adjuvant. The same findings were reported in some of the Iranian trials (Sharifi et al. 1998, Momeni et al. 1999).

\section{Concluding remarks}

Despite few negative aspects of KLV and BCG applications solely or in accompany in different clinical forms of leishmaniasis (Noazin et al. 2009, Jamshidi et al. 2011), acceptable efficacy of KLV + BCG is indicated here on reduction of lesion sizes, proliferation of amastigotes in MQs and visceralization of parasites in spleen and lymph nodes, which is in agreement with several published reports (Kamil et al. 2003, Mohebali et al. 2004, Khalil et al. 2006, Michel et al. 2006, Keshavarz Valian et al. 2008, Pereira et al. 2009, Nateghi Rostami et al. 2010, Soudi et al. 2011). It is indicated that KLV/BCG may have the ability to modulate host immune responses against parasite proliferation and to reduce pathophysiology of infection. Infection with $L$. major in mice indicates that a protective immune response is achieved when Th1 cells are developed. Thus, adoptive or vaccine-induced protection against leishmaniasis is largely dependent on cell- 

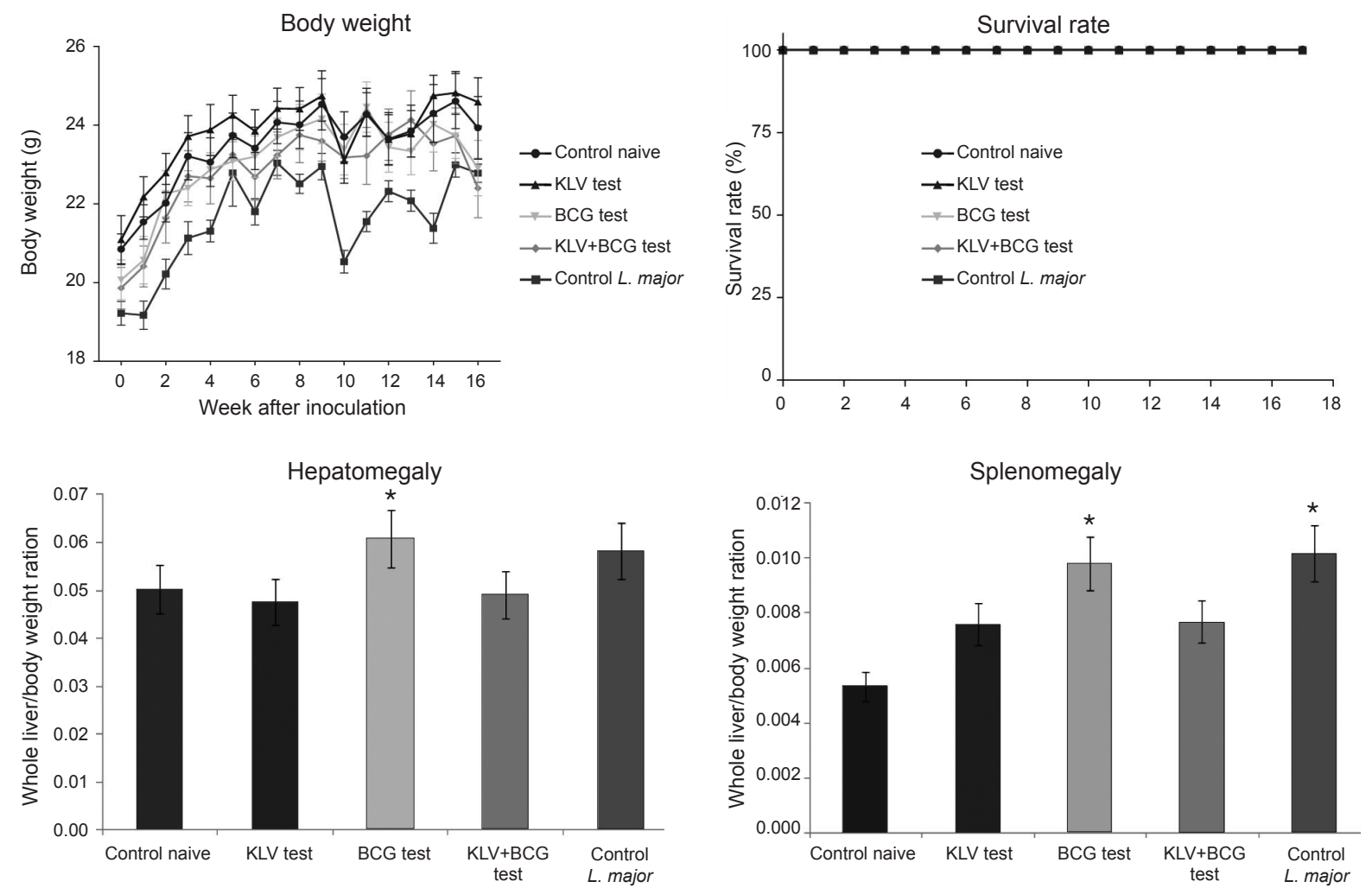

Fig. 4. Influence of KLV and BCG on pathophysiology. Pathophysiological parameters including body weights, survival rates and degrees of hepatosplenomegaly were assessed in experimental groups as described in the Materials and methods. The results expressed as mean \pm SE. Significance of difference $(\star \mathrm{P}<0.05)$ was determined by ANOVA test using Graph Pad Prism and Microsoft Excel 2007 ( $=10$ mice/group).

mediated immunity and cytokine production (Pearson et al. 1983). Therefore, complementary investigations may be suggested on KLV and BCG using different doses and concentrations, the route of inoculation, different Leishmania strains and host species.
Acknowledgments. This study was supported by the Department of Parasitology, in collaboration with the Department of Biochemistry, at the Pasteur Institute of Iran. Some results presented in this manuscript form part of a MSc thesis (Seyedeh Parisa Jafary) from the Islamic Azad University of Oloum Tahghighat, Tehran, Iran under the first author supervision.

\section{REFERENCES}

Abdelhak S., Louzir H., Timm J., Blel L., Benlasfar Z., Lagranderie M., Gheorghiu M., Dellagi K., Gicquel B. 1995: Recombinant BCG expressing the Leishmania surface antigen Gp63 induces protective immunity against Leishmania major infection in BALB/c mice. Microbiology 141: 1585-1592.

Amaral V.F., Teva A., Oliveira-Neto M.P., Silva A.J., Pereira M.S., Cupolillo E., Porrozzi R., Coutinho S.G., Pirmez C., Beverley S.M., GRIMALDi G. JR. 2002: Study of the safety, immunogenicity and efficacy of attenuated and killed Leishmania (Leishmania) major vaccines in a rhesus monkey (Macaca mulatta) model of the human disease. Mem. Inst. Oswaldo Cruz 97: 1041-1048.

Antunes C.M.F., Mayrink W., Magalhaes P.A., Costa C.A., Melo M.N., Dias M., Michalick M.S., William P., Lima A.O., Vieira J.B. 1986: Controlled field trials of a vaccine against new world cutaneous leishmaniasis. Int. J. Epidemiol. 15: 572-580.

Castes M., Blackwell J., Trujillo D., Formica S., Cabrera M., Zorrilla G., Rodas A., Castellanos P.L., Convit J. 1994: Immune response in healthy volunteers vaccinated with killed leishmani- al promastigotes plus BCG 1: skin test reactivity, T-cell proliferation, and interferon-gamma production. Vaccine 12: 1041-1051.

Convit J., Castellanos P.L., Rondon A., Pinardi M.E., Ulrich M., Castes M., Bloom B., Garcia L. 1987: Immunotherapy versus chemotherapy in localized cutaneous leishmaniasis. Lancet 1 : 401-405.

El-On J., Witztum A., Schnur L.F. 1986: Protection of Guinea pigs against cutaneous leishmaniasis by combined infection and chemotherapy. Inf. Immun. 51: 704-706.

Erel O., Kocyigit A., Bulut V., Gurel M.S. 1999: Reactive nitrogen and oxygen intermediates in patients with cutaneous leishmaniasis. Mem. Inst. Oswaldo Cruz 94: 179-183.

Garg R., Dube A. 2006: Animal models for vaccine studies for visceral leishmaniasis. Ind. J. Med. Res. 123: 439-454.

Green M.S., Kark J.D., Witztum E., Greenblatt C.L., Sira D.T. 1983: Frozen stored L. tropica vaccine: the effect of dose, route for administration and storage on the evolution of clinical lesion. Two field trials in the Israel defense forces. Trans. R. Soc. Trop. Med. Hyg. 77: 152-159. 
Jamshidi Sh., Avizeh R., Mohebali M., BoKaie S. 2011: Immunotherapy using autoclaved L. major antigens and M. vaccae with Meglumine antimoniate, for the treatment of experimental canine visceral leishmaniasis. Iran. J. Parasitol. 6: 26-34.

Kamil A.A., Khalil E.A., Musa A.M., Modabber F., Mukhtar M.M., Ibrahim M.E., Zijlstra E.E., SACKs D., Smith P.G., Zicker F., El-Hassan A.M. 2003: Alum-precipitated autoclaved Leishmania major plus bacille Calmette-Guérin, a candidate vaccine for visceral leishmaniasis: safety, skin-delayed type hypersensitivity response and dose finding in healthy volunteers. Trans. R. Soc. Trop. Med. Hyg. 97: 365-368.

Keshavarz Valian H., Khoshabe Abdollah Kenedy L., Nateghi Rostami M., Miramin Mohammadi A., Khamesipour A. 2008: Role of Mycobacterium vaccae in the protection induced by first generation Leishmania vaccine against murine model of leishmaniasis. Parasitol. Res. 103: 21-28.

Khalil E.A., Musa A.M., Modabber F., El-Hassan A.M. 2006: Safety and immunogenicity of a candidate vaccine for visceral leishmaniasis (Alum-precipitated autoclaved Leishmania major + BCG) in children: an extended phase II study. Ann. Trop. Paediatr. 26: 357-361.

Klaus S.N., Frankenburg S., Ingber A. 1999: Epidemiology of cutaneous leishmaniasis. Clin. Dermatol. 17: 257-260.

Kobets T., Grekov I., Lipoldová M. 2012: Leishmaniasis: prevention, parasite detection and treatment. Curr. Med. Chem. 19: 1443-1474.

Latifynia A., Mohaghegh Hazrati S. 2008: Safety and toxicity of a new formulated Leishmania major preliminary vaccine in animal model Balb/c and small white conventional laboratory mice. Türk. Parazitol. Dergisi 32: 103-108.

Manson-Bahr P.E.C., Apted F.I. 1983: Manson's Tropical Diseases, 18th edition. Bailliere Tindall, London, $843 \mathrm{pp}$.

Mayrink W., da Costa C.A., Magalhaes P.A., Melo M.N., Dias M., Lima A.O., Michalick M.S., William P. 1979: A field trial of a vaccine against American dermal leishmaniasis. Trans. R. Soc. Trop. Med. Hyg. 73: 385-387.

Mayrink W., Williams P., da Costa C.A., Magalhães P.A., Melo M.N., Dias M., Oliveira Lima A., Michalick M.S., Ferreira Carvalho E., Barros G.C. 1985: An experimental vaccine against American dermal leishmaniasis: experience in the state of Espirito Santo, Brazil. Ann. Trop. Med. Parasitol. 79: 259-269.

Michel M.Y., Fathy F.M., Hegazy E.H., Hussein E.D., Eissa M.M., SAID D.E. 2006: The adjuvant effects of IL-12 and BCG on autoclaved Leishmania major vaccine in experimental cutaneous leishmaniasis. J. Egypt. Soc. Parasitol. 36: 159-176.

Mohebali M., Javadian E.H., Hashemi-Fesharki R., Mohammad Zadeh M., Nadim A., Tahvildar-Bidruni G.H., Mesdaghim A. 1995: Trial of a nonliving crude vaccine against zoonotic cutaneous leishmaniasis. Med. J. Islamic Rep. Iran 8: 211-215.

Mohebali M., Khamesipour A., Mobedi I., Zarei Z., Hashemi-FesHARKI R. 2004: Double-blind randomized efficacy field trial of alum precipitated autoclaved Leishmania major vaccine mixed with BCG against canine visceral leishmaniasis in MeshkinShahr district, I.R. Iran. Vaccine 22: 4097-4100.

Momeni A.Z., Jalayer T., Emamjomeh M., Khamesipour A., Zicker F., Ghassemi R.L., Dowlati Y., Sharifi I., Aminjavaheri M., Shafiei A., Alimohammadian M.H., Hashemi-Fesharki R., NasSeri K., Godal T., Smith P.G., Modabber F. 1999: A randomized double-blind, controlled trial of killed L. major vaccine plus BCG against zoonotic cutaneous leishmaniasis in Iran. Vaccine 17: $466-472$

Nadim A., Javadian E. 1988: Leishmanization in the Islamic Republic of Iran. In: B.C. Walton, P.M. Wijeyaratna and F. Modabber
(Eds.), Research on Control Strategies for Leishmaniasis. International Development Research Centre, Toronto, pp. 336-339.

Nahrevanian H., Farahmand M., Aghighi Z., Assmar M., AmirkhaNI A. 2007: Pharmacological evaluation of anti-leishmanial activity by in vivo nitric oxide modulation in Balb/c mice infected with Leishmania major MRHO/IR/75/ER: an Iranian strain of cutaneous leishmaniasis. Exp. Parasitol. 116: 233-240.

Nateghi Rostami M., Keshavarz H., Khamesipour A. 2010: Immune response of $\mathrm{BALB} / \mathrm{c}$ mice against an experimental vaccine of Alum precipitated autoclaved Leishmania major (Alum-ALM) mixed with BCG or Mycobacterium vaccae. Trop. Biomed. 27: 89-102.

Noazin S., Khamesipour A., Moulton L.H., Tanner M., Nasseri K., Modabber F., Sharifi I., Khalil E.A., Bernal I.D., Antunes C.M., Sмiтн P.G. 2009: Efficacy of killed whole-parasite vaccines in the prevention of leishmaniasis: a meta-analysis. Vaccine 27: 4747-4753.

Pearson R.D., Wheeler D.A., Harrison L.H., Kay H.D. 1983: The immunobiology of leishmaniasis. Rev. Infect. Dis. 5: 907-927.

Pereira L.I., Dorta M.L., Pereira A.J., Bastos R.P., Oliveira M.A., Pinto S.A., Galdino H. Jr., Mayrink W., Barcelos W., Toledo V.P., Lima G.M., Ribeiro-Dias F. 2009: Increase of NK cells and proinflammatory monocytes are associated with the clinical improvement of diffuse cutaneous leishmaniasis after immunochemotherapy with BCG/Leishmania antigens. Am. J. Trop. Med. Hyg. 81: 378-383.

Roberts W.L., McMurray W.J., Rainey P.M. 1998: Characterization of the antimonial antileishmanial agent meglumine antimoniate (Glucantime). Antimicrob. Agents Chemother. 42: 1076-1082.

Salehizadeh E., Nahrvevanian H., Farahmand M., Hajihosseini R., SAghiRI R., Khalili G. 2011: In vivo application of killed Leishmania vaccine and Imiquimod as adjuvant in Balb/c mice infected with Leishmania major MRHO/IR/75/ER as Iranian strain. Res. J. Parasitol. 6: 116-126.

Sсотт P. 2005: Immunologic memory in cutaneous leishmaniasis. Cell Microbiol. 7: 1707-1713.

Sharifi I., Fekri A.R., Aflatonian M.R., Khamesipour A., Nadim A., Mousavi M.R., Momeni A.Z., Dowlati Y., Godal T., Zicker F., Sмith P.G., Modabber F. 1998: Randomised vaccine trial of single dose of killed Leishmania major plus BCG against anthroponotic cutaneous leishmaniasis in Bam, Iran. Lancet 351: $1540-1543$.

SMRKovSKi L.L., LARSON C.L. 1977a: Antigenic cross-reactivity between Mycobacterium bovis (BCG) and Leishmania donovani. Infect. Immun. 18: 561-562.

SMRKOVSKI L.L., LARSON C.L. 1977b: Effect of treatment with BCG on the course of visceral leishmaniasis in BALB/c mice. Infect. Immun. 16: 249-257.

Soudi S., Hosseini A.Z., Hashemi S.M. 2011: Co-administration of rectal BCG and autoclaved Leishmania major induce protection in susceptible BALB/c mice. Parasite Immunol. 33: 561-571.

Stefani M.M., Müller I., LouIs J. 1993: Leishmania major infection in BALB/c mice: protection or exacerbation by treatment with different doses of BCG. Res. Immunol. 144: 233-243.

UNDP/WORLD BANK/WHO 1997: Tropical disease research: progress 1995-1996, Thirteenth Programme Report of the UNDP/World Bank/WHO, Special Programme for Research \& Training in Tropical Diseases, 149 pp. (http://www.who.int/tdr/ publications/about-tdr/progress-reports/progress-report-95-96/ en/index.html)

Weintraub J., Weinbaum F.I. 1977: The effect of BCG on experimental cutaneous leishmaniasis in mice. J. Immunol. 118: 2288 2290. 